\title{
Methods of reducing power losses in distribution systems
}

\author{
Yakov Wilms ${ }^{1, *}$, Sergey Fedorovich, and Nikolai A. Kachalov \\ ${ }^{1}$ National Research Tomsk Polytechnic University, 634050 Tomsk Russia
}

\begin{abstract}
The object of research is plot of an urban electricity distribution network of $10 / 0.4 \mathrm{kV}$ of Tomsk city. The purpose of work - research and development of scientific and technical solutions to optimize the plot operation modes of an urban power supply system on the basis of modern software and hardware. The study found ways to reduce the unbalance of currents and voltages. Applications: urban and rural distribution networks 10/0.4 kV. Economic efficiency: the measures proposed can reduce the power loss. In the course of the research calculation and analyzing of the operation mode in the programs were carried out.
\end{abstract}

\section{Introduction}

Russia is one of the most energy-intensive economies in the world. This is due to climatic conditions, as well as the location of industry and population in its vast territory. According to the conducted research there is a significant potential for energy saving in Russia and this will reduce electricity use by half. There is the problem inefficient use of electricity and energy equipment in distribution networks as a result of unbalanced phase load, etc.

Thus, the strategic aim of this research is determination of the optimal operating mode in distribution networks and development of measures to reduce power losses.

There are different methods for reduce power losses and mainly these methods are grouped in three groups: organizational, technical and methods for improvement an accounting electricity system. Organizational events consist of:

1. finding of disjunction locations with duplicate electric power supply;

2. reducing an unbalanced phase load;

3. optimization of loading of power transformers.

Technical events mean using special equipment, for example: to reduce power losses, a shunt capacitor bank can be installed on distribution primary feeders, also to reduce an unbalanced phase load; a harmonic filter can be installed. Methods for improving an accounting electricity system is due with an application of automatic counting systems of electricity and an application of high accuracy level electric meters.

The object of research is plot an urban electricity distribution network of $10 / 0.4 \mathrm{kV}$ in Tomsk city. The main disadvantage of this part is inefficient equipment loading (load factor; most of transformers have load factor below than $45 \%$ ). Since this system is in working order, consideration of organizational events is preferable because they don't need reconstruction

\footnotetext{
* Corresponding author: yawilms@yandex.ru
} 
and also they don't demand a significant investment. In addition, in the work of I.I. Elfimov [1] considered the possibility of installing capacitor banks and replacing transformers with zigzag transformers. As a result, reduction of the current asymmetry and power losses were reported, but this result is not significantly.

This paper provides the next events:

1. finding of disjunction locations with duplicate electric power supply;

2. disconnection of equipment for minimization power losses.

\section{Modes of operation of electrical networks}

The mode of operation of electrical networks is the state of the system, which is characterized by indicators that quantify its operation. The operating parameters include: values of power, voltage, current, frequency, etc.

The operating modes of electrical networks are divided into steady-state and transient ones, connected with the studies of dynamic stability [6]. In turn, the steady-state modes are comprised of:

1. normal mode;

2. emergency state;

3. forced state [7].

A Load curve changes depending on the seasons of the year, in connection with it there are the maximum load mode (winter maximum) and the minimum load mode (summer minimum).

Calculations of the modes of the electric network are performed to determine:

1. loading of network elements, matching the network capacity to expected power flows;

2. cross-sections of wires and power cables of transformers and autotransformers;

3. the level of voltage in the nodes of the electrical network and measures ensuring the maintenance of voltage within the permissible limits;

4. loss of power and electrical energy;

5. the capacity of the electrical network, etc.

\section{Finding of disjunction locations with duplicate electric power supply}

The existing methods of selecting the optimal places for disjunction electric network can be divided into 2 groups. The methods of the first group imply a completely closed position of circuit breakers at first, but methods of the second group are completely opened originally. Then, circuit breakers operate (opening/closing) occur until minimum power losses develop in the network. There were considered both groups, but most visible was the first group method. In general form, the algorithm of the first group method is shown in figure 1. 


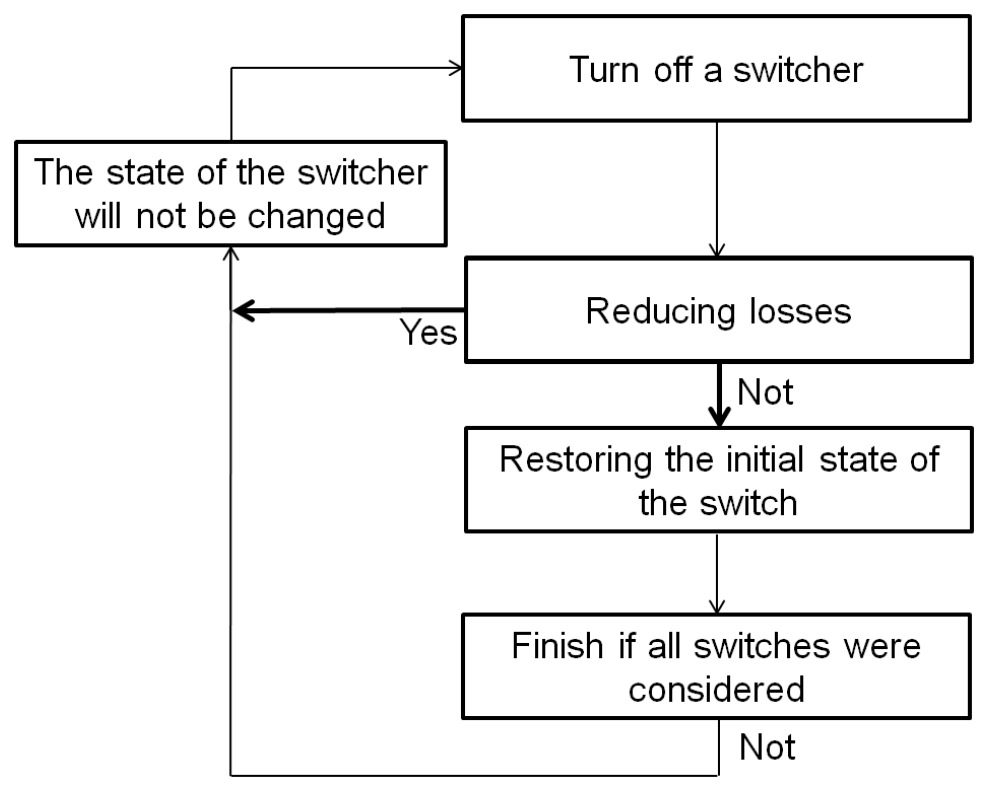

Fig. 1. Finding of disjunction locations algorithm.

Both of these methods have the same results, but the use of them changed nothing for this plot, because the resulting state of the network resembled the original state.

\section{Disconnection part of equipment (Switching off part of the equipment)}

Such as the main feature of this plot is the low load of power grid equipment, it is worth considering the possibility of its disconnection. There are transformers and cable lines in this plot.

There are no-load losses (rotational losses) and load losses (variable losses) in transformers, and the greater the power of the transformer, the greater the no-load losses. Power losses are calculated by the formula (for one transformer):

$$
\Delta P_{\text {trans }}=\Delta P_{N-L L}+\Delta P_{L L} \cdot \frac{S^{2}}{S_{\text {nom }}^{2}}
$$

Therefore, disconnecting one of the two transformers on the substation will lead to a reduction of no-load losses. For two transformers, power losses are calculated by the formula (Parallel):

$$
\Delta P_{\text {trans }}=\Delta P_{N-L L} \cdot n+\frac{1}{n} \cdot \Delta P_{L L} \cdot \frac{S^{2}}{S_{n o m}^{2}}
$$

In that load factors of each transformer on substations are different, there is need to examine parallel and separate modes of the transformers work. To determine the value of the load power at which the operation of one transformer is economically more feasible than the parallel operation of two transformers we can use the following formula:

$$
S_{\text {optimal }} \geq S_{\text {nom }} \cdot \sqrt{\frac{2 \cdot \Delta P_{N-L L}}{\Delta P_{L L}}}
$$




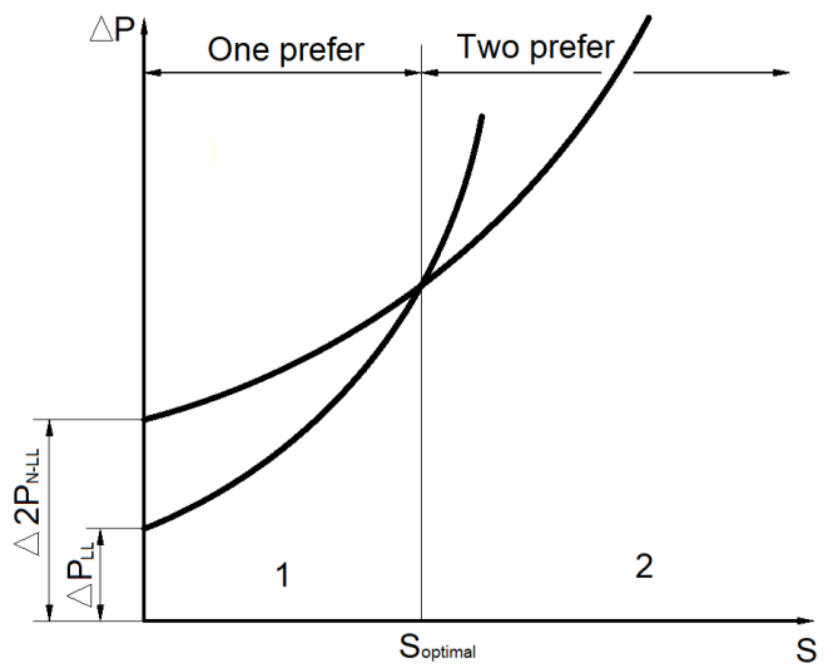

Fig. 2. Suitable operating modes of transformers.

According to the calculation results, usage of one transformer is preferred for all substations (figure 2, plot 1). However, the simulation of this mode showed the opposite, because cable lines have only load losses, and for disconnection of the first transformer followed the disconnection the first cable line on the substation, and also loading of the second cable line was accompanied by an increase in losses. In the issue of the power losses in transformers decreased and the power losses in cable lines increased by the same value. Nevertheless there was a stable voltage profile (level voltage) originally, but a working mode with disconnected second transformers on a substation have an under voltage level, and therefore disconnection parts of energy equipment is ineffective for this plot of distribution network.

There is a problem to reduce losses in this plot of power distribution power system, but this was considering the on-peak (LP winter case) conditions and this case is idealized. It is necessary to consider the real case - daily load curve. As the result of that investigation found (figure 3), there is a way to reduce power losses with increased load power (specific losses). Proceeding from the aforesaid the next part of our investigation is putting the system out of balance - modeling of load power increase.

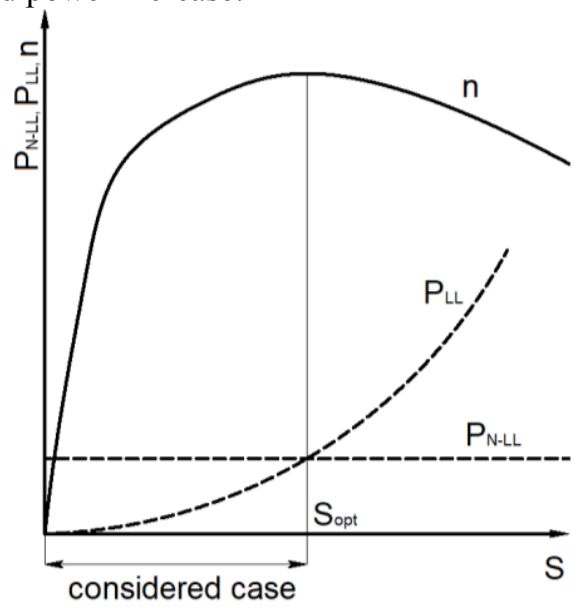

Fig. 3. Curve of efficiency factor $(n)$ with dependence of no-load losses $\left(\mathrm{P}_{\mathrm{N}-\mathrm{LL}}\right)$ and load losses $\left(\mathrm{P}_{\mathrm{LL}}\right)$. 


\section{References}

1. I.I. Elfimov, E. A. Shutov, Optimization of operating modes of electrical networks (TPU, Tomsk, 2016)

2. G.N. Klimova, Energy saving on industrial enterprises (TPU, Tomsk, 2014)

3. D.E. Dulepov, T.E. Tyundina, Vestnik NGIEI, 4 (2015)

4. M.I. Fursanov, Energetika, 60 (2014)

5. A.L. Trushnikov, V.N. Radkevich, GGTU, 1 (2006)

6. E.S. Tarasova , I.B. Daminov, EPJ Web Conf. 110 (2016)

7. E.S. Tarasova, I.B. Daminov, MATEC Web Conf. 37 (2015) 